\title{
Osseous metastases and ovarian cancer
}

\author{
Monica Cirstoiu* **, Alexandru Baros* **, Florina Paulet* **, Delia Gradinaru-Fometescu**, \\ Bogdan Șerban****, Bogdan Cretu***, Cătălin Cirstoiu**** \\ *"Carol Davila" University of Medicine and Pharmacy, Bucharest, Romania \\ **Department of Obstetrics and Gynaecology, University Emergency Hospital, Bucharest, Romania \\ ***Department of Orthopaedics and Traumatology, University Emergency Hospital, \\ Bucharest, Romania
}

Correspondence to: Alexandru Baros, MD, PhD, Department of Obstetrics and Gynaecology, University Emergency Hospital, Bucharest, 169 Splaiul Independenței Street, District 5, Bucharest, Romania,

Phone: 02131805 19, int. 521, Mobile phone: +40721278 747,

E-mail: alexandrubaros@yahoo.com

\begin{abstract}
This article analyses a series of 22 confirmed cases of ovarian cancer throughout a period of 14 months (January 2018 - February 2019), in which we assessed the incidence of osseous metastases, in correlation with their histological features. This retrospective study, based on medical imaging techniques, demonstrates that bone metastases are not uncommon in ovarian cancer.
\end{abstract}

Keywords: osseous, metastases, ovarian, carcinoma, incidence

\section{Introduction}

Ovarian cancer is the 8th most common type of neoplasia in women. In 2018, almost 300,000 new cases were registered [1] worldwide. Romania is among the top 25 countries with the highest rates of ovarian cancer in 2018 (number 20), with an agestandardized rate of 1,4 per 100,000 [1].

Bone metastases are one of the first signs of disseminated disease in cancer patients [2], and typically indicate a short-term prognosis in cancer patients. However, osseous metastasis from ovarian cancer is rare, but the increasing pathological stage of ovarian cancer may contribute to the risk of bone metastasis (especially in the cases with lung or lymphatic dissemination) [3].

Bone is a common metastatic site and bone metastases most commonly affect the axial skeleton, thus, bone metastases represent a major cause of morbidity in the patients diagnosed with advanced-stage malignant diseases. Morbidities, like pathological fractures and spinal cord compression/ paralysis, cause impairment in activities of daily life and affect prognosis because of deterioration of the affected patient's general condition and discontinuation of treatment for the primary disease. Approximately $70 \%$ of all malignant bone tumors are metastatic in origin. The incidence varies according to the type of primary neoplasm and the duration of the disease [4]. 


\section{Methods and materials}

In a review of 263 cases admitted with the diagnosis of ovarian tumor over the last 14 months in the Department of Obstetrics and Gynaecology, University Emergency Hospital, in Bucharest, 22 were later confirmed as ovarian cancer.

Most of the ovarian tumors proved to be endometriotic cysts (19 cases), cystadenofibroma of the ovary (mucinous included, 34 cases), dermoid cysts (18 cases).

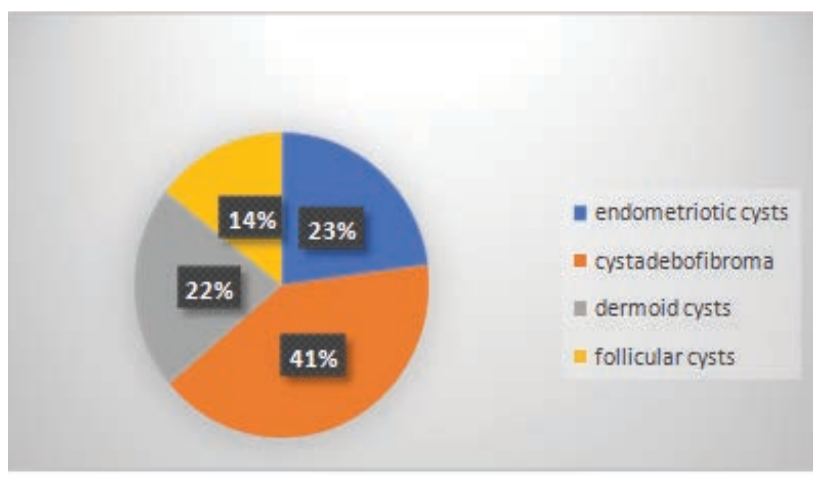

Fig. 1 Benign ovarian tumors

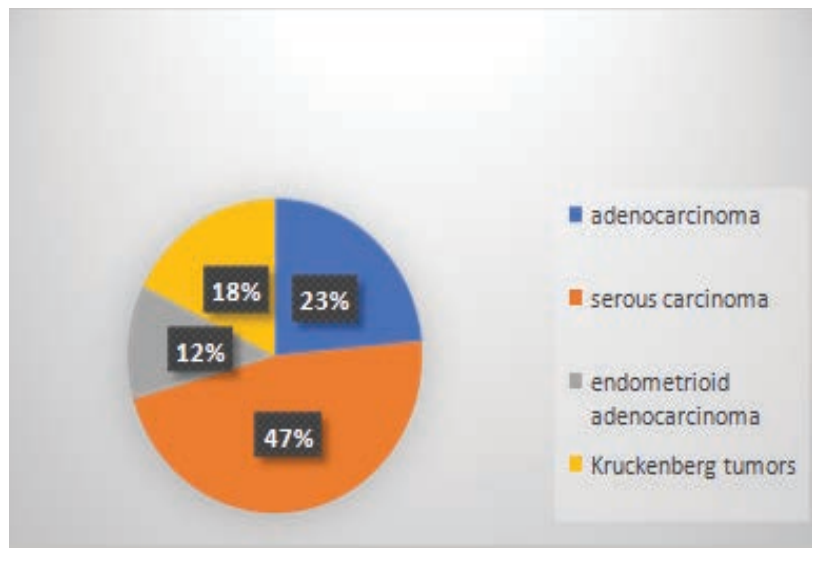

Fig. 2 Malignant and borderline ovarian tumors

\section{Results}

Out of the 22 cases of ovarian cancer, 4 managed to disseminate to the bone. It was also observed that pulmonary, hepatic, and peritoneal metastases were registered.
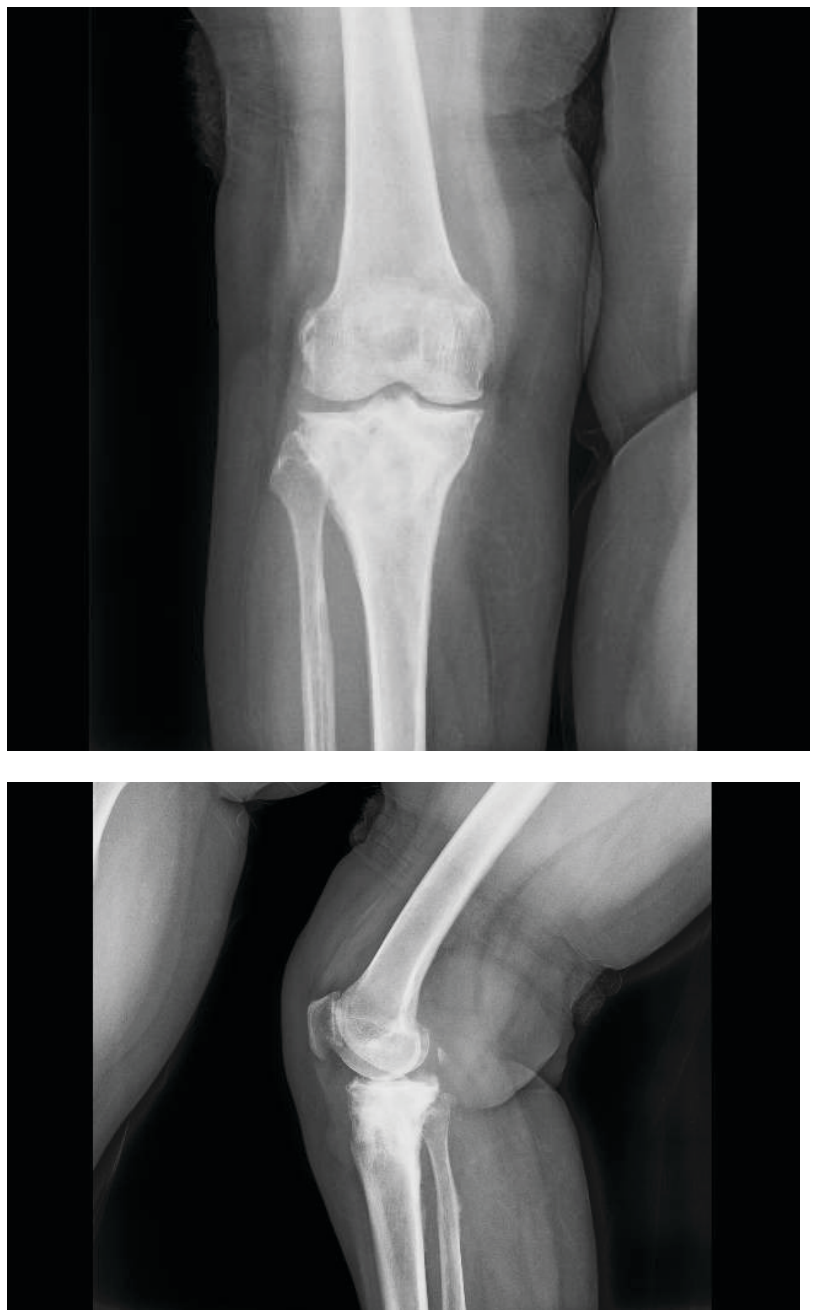

Fig. 3 X-ray image of a proximal tibia with a blastic lesion secondary to a squamous cell carcinoma
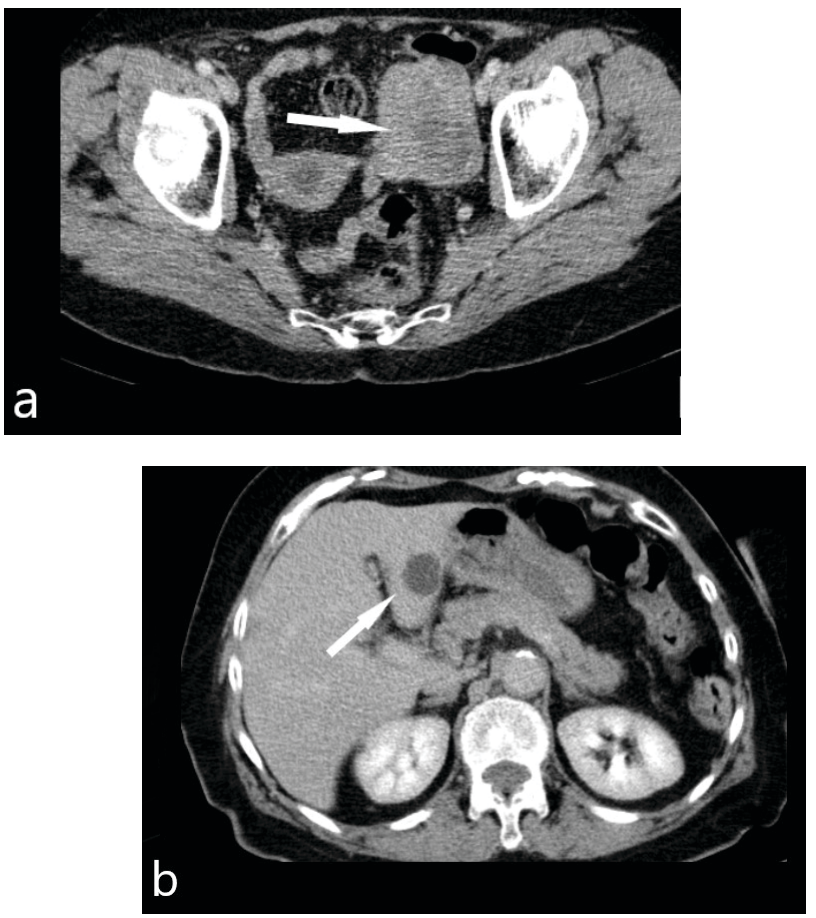

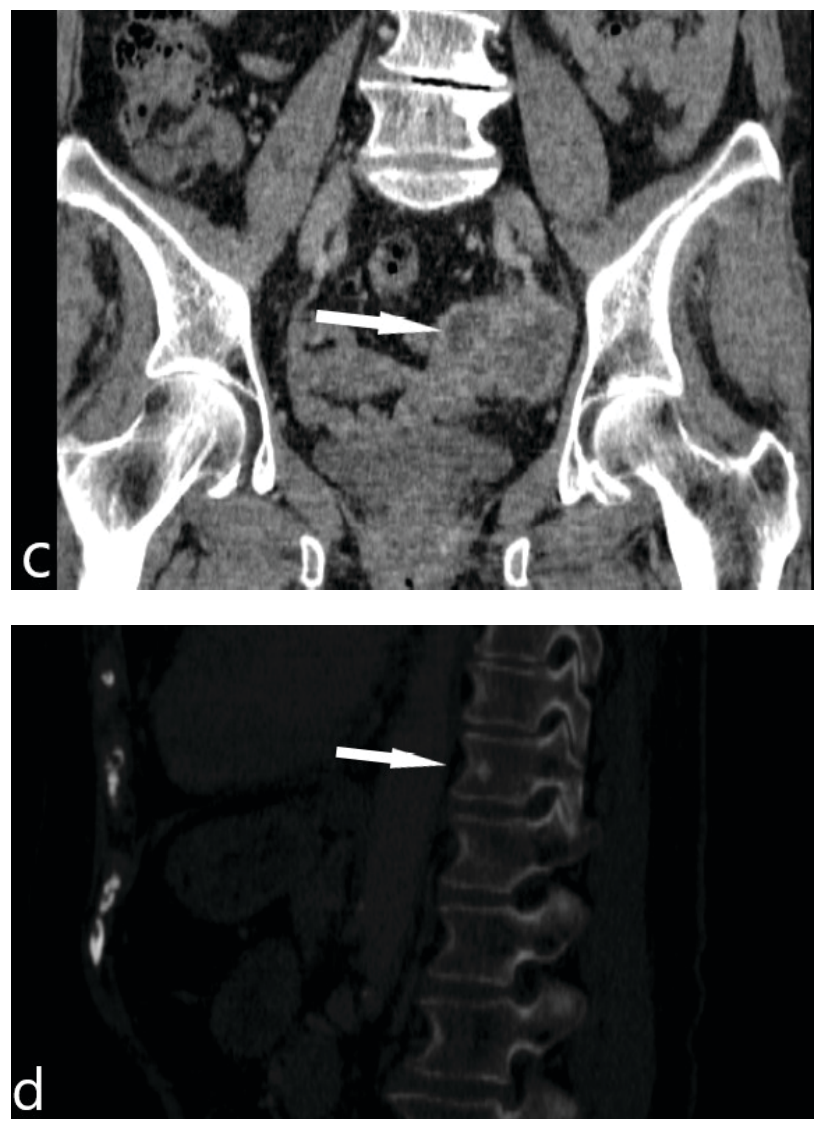

Fig. 4 CT scan of the abdomen and pelvis, with contrast: a, c: axial (a) and coronal (c) sections that highlight a left hemi pelvic parauterine polilobular mass, of mixed component (both tissular and fluid) - histopathologically confirmed as high-grade serous ovarian carcinoma; $b$ : axial section of the superior abdominal cavity, highlighting a hypodense hepatic lesion, well-defined, located in the IIIrd segment of the liver (metastasis); d: sagittal section of the thoracolumbar spine examined in, highlighting an osteocondensation lesion located in the T11 vertebral body (suspected metastasis)

\section{Conclusion}

The risk of developing ovarian cancer rises as a country becomes more developed, and as areas become more urbanized. This means the three drivers of cancer burden (increasing populations, increased longevity, and increases risk because of environmental factors) will mean ovarian cancer becomes even more an issue in developed, and less developed countries. There are also many other variants affecting risk and mortality rates, including ethnicity, tumor types, and age profiles.

Developments in medical imaging offer new hope in terms of targeted treatments, and primary prevention, improving greatly the management of the case and subsequently the quality of life for the patient [5].

\section{Conflict of Interest statements}

Authors state no conflict of interest.

\section{Informed Consent and Human and Animal}

\section{Rights statements}

Informed consent has been obtained from all individuals included in this study.

\section{Authorization for the use of human subjects}

Ethical approval: The research related to human use complies with all the relevant national regulations, institutional policies, is in accordance with the tenets of the Helsinki Declaration, and has been approved by the authors' institutional review board or equivalent commitee.

\section{References}

1. The online GLOBOCAN 2018 database is accessible at http://gco.iarc.fr/, as part of IARC's Global Cancer Observatory. http://gco.iarc.fr/today/fact-sheets-cancers.

2. Nielsen OS, Munro AJ, Tannock IF. Bone metastases: pathophysiology and management policy. Journal of Clinical Oncology. 1991; 9(3),509-524.

3. Zhang M, Sun J. Bone metastasis from ovarian cancer. Clinical analysis of 26 cases. Saudi Medical Journal. 2013; 34(12),1270-1273.

4. Kaku T, Ogawa S, Kawano Y, Ohishi Y, Kobayashi H, Hirakawa T, Nakano H. Histological classification of ovarian cancer. Medical Electron Microscopy. 2003; 36(1),917.

5. Tempany CM, Zou KH, Silverman SG, Brown DL, Kurtz AB, McNeil BJ. Staging of advanced ovarian cancer: comparison of imaging modalities-report from the Radiological Diagnostic Oncology Group. Radiology. 2000; 215(3),761-767. 